\title{
Epidemiology and outcomes of community-acquired and hospital-acquired acute kidney injury in children and adolescents
}

\author{
Chien-Ning Hsu ${ }^{1,2}$, Hsiao-Ling Chen ${ }^{1}$ and You-Lin Tain ${ }^{3}$
}

BACKGROUND: Hospital-acquired acute kidney injury (HAAKI) is associated with an increased risk of childhood mortality; however, only a few studies have addressed communityacquired AKI (CA-AKI).

METHODS: AKI network classification was used to assess CAand HA-AKI, 2010-2014. Patients with CA-AKI who were admitted to an inpatient setting were categorized as CAA-AKI. CANA-AKI was for CA-AKI not admitted to inpatient care. Epidemiology, factors associated with AKI, and in-hospital outcomes were assessed for variation.

RESULTS: Prevalence of CANA-AKI was 4/1,000 outpatient visits, 17/1,000 hospital admissions for CAA-AKl, and 9.69/1,000 hospital admissions for HA-AKI. Mortality was higher among AKI patients (HA-AKI, 13.64\%; CAA-AKI, 3.7\%) than in no-AKI patients $(0.57 \%)$. Patients with AKI and those with severe stages of AKI resulted in an increase in health-care service utilization (both $P<0.001$ ). Prior renal disease and recent hospitalization were associated with pediatric AKI in both outpatient and inpatient settings. Hematological malignancies, congenital anomalies, circulatory disease, and nephrotoxic medication use were associated with AKI, although the extent of associations varied slightly by setting.

CONCLUSION: Increasing incidence of AKI in the community emphasizes the need for an increased awareness of AKI among health professionals to identify at-risk children and monitor SCr, so that modifiable risk factors can be managed.

T he development of acute kidney injury (AKI) during hospitalization (hospital-acquired AKI; HA-AKI) is a widely recognized complication that is associated with a substantially increased risk of mortality in both critically (13 ) and non-critically $(4,5)$ ill-hospitalized children. Globally, the impact of AKI on patient outcomes and health-care service utilization is significant in both adult and children populations. Recent data from the Assessment of Worldwide Acute Kidney Injury, Renal Angina, and Epidemiology (AWARE) (6), a multinational, cross-sectional study, reinforce the importance of monitoring the development of AKI and adequate management in critically ill children, as in the case of adults in the AKI-EPI (Epidemiologic Prospective Investigation) study (7). AKI is associated with an approximately twofold increase in the risk of 28-day morality and an increase in health-care service utilization (i.e., mechanical ventilation, renal replacement therapy, and length of stay $(L O S)$ ) in a pediatric intensive care setting (6). Further, some patients who do not regain renal function at the time of hospital discharge are at risk of developing chronic kidney disease $(\mathrm{CKD})$ in young adulthood $(8,9)$.

AKI is both a community- and hospital-acquired condition. Patients who present to hospitals with reduced urine output (UO) and/or glomerular filtration rate are thought to have community-acquired AKI (CA-AKI). Our understanding of the epidemiology of CA-AKI has been limited by the lack of uniformity in the definition of AKI, and recent reviews suggest that there are etiological and geographical differences in the characteristics of CA-AKI in different regions of the world $(10,11)$.

A study examined three recent consensual definitions of AKI according to creatinine-based classifications, i.e., pediatric RIFLE, AKI Network (AKIN), and Kidney Disease Improving Global Outcomes (KDIGO) criteria, and concluded that all have excellent discriminative capability for patient outcome and LOS in hospitalized children (12). However, the utility of these recent AKI criteria for the diagnosis of CA-AKI in children has not been well studied. AKIN criteria are considered most applicable in a retrospective research using electronic medical records; the AKIN criteria do not require the application of body height or baseline serum creatinine levels that are not routinely determined in general practice (Supplementary Table S1 online). The AKIN criteria are particularly important for children and adolescents in outpatient settings who do not undergo regular blood tests to determine SCr levels. The limitations of using the KDIGO creatinine-based criteria in clinical practice, such as the assumption that patients undergo at least two SCr measurements in a week, thereby leading to

\footnotetext{
${ }^{1}$ Department of Pharmacy, Kaohsiung Chang Gung Memorial Hospital, Kaohsiung, Taiwan; ${ }^{2}$ School of Pharmacy, Kaohsiung Medical University, Kaohsiung, Taiwan; ${ }^{3}$ Division of Pediatric Nephrology, Kaohsiung Chang Gung Memorial Hospital and Chang Gung University, College of Medicine, Kaohsiung, Taiwan. Correspondence: You-Lin Tain (tainyl@hotmail.com)

Received 4 January 2017; accepted 29 September 2017; advance online publication 3 January 2018. doi:10.1038/pr.2017.262
} 


\section{Epidemiology and outcomes of pediatric AKI $\mid$ Articles}

the misclassification of patients without AKI, have been described in previous studies (13).

In order to achieve early identification, proper management, and prevention of mortality associated with AKI in general practice settings, it is imperative to understand the epidemiological features, risk factors, and outcomes in pediatric AKI patients. By using the diagnostic definition of AKI and the AKIN staging system (14), we evaluated the incidence and prevalence of AKI, mortality, and LOS in three AKI groups in a large pediatric cohort in Taiwan: CANA-AKI patients who were not hospitalized, CA-AKI patients who were hospitalized (CAA-AKI), and HA-AKI patients. Further, we evaluated the associations between patient characteristics and the development of AKI in outpatient and inpatient settings.

\section{METHODS}

\section{Data Sources and Study Population}

Before data collection, the study was approved by the Institutional Review Board and the Ethics Committee of Chang Gung Medical Foundation, Taoyun, Taiwan. The need for obtaining informed patient consent was waived because of the retrospective study design, and data were extracted anonymously without distinguishing identifiers.

We conducted a cross-sectional study using EMRs to assess the epidemiological features and outcomes of AKI in children admitted to Chang Gung Memorial Hospital, Taiwan, between 1 January 2010 and 31 December 2014. The inclusion criterion was that the patients should be 28 days to 18 years old at the time of admission. Exclusion criteria included pre-existing renal replacement therapy (dialysis and renal transplantation) and lack of SCr measurements at the index, baseline, and $\geq 1$ follow-up points during hospitalization (Figure 1). Baseline creatinine was defined as the most recent creatinine measurement obtained in the 3 months before admission, inclusive of the day of admission.

\section{AKI Definition}

AKI cases were categorized according to the AKIN stages $1-3$ and were based on the baseline and index SCr measurements (Table 1). If the index SCr value at admission increased by $0.3 \mathrm{mg} / \mathrm{dl}$ from the baseline within a 48-h period, the AKI episode was categorized as stage 1. For CA-AKI cases, baseline SCr was defined as the most recent measurement of SCr within the 3 months before the index $\mathrm{SCr}$ measurement at admission. If more than one measurement was carried out in a day during the baseline period, the earliest value was used. In the case of hospitalized patients with CA-AKI who were transferred from the emergency department or ambulatory settings (CAA-AKI), the baseline, and index SCr values were the same as those for CANA-AKI. The HA-AKI group comprised patients for whom the highest measured SCr values during hospitalization increased $\geq 1.5$-fold above the admission value (peak/index SCr $\geq 1.5$ ).

\section{Clinical Characteristics}

Demographic data included age at the time of admission and sex as recorded in the EMRs. Clinical characteristics included prior use of drugs that could cause adverse reactions in patients with impaired renal function; pre-existing CKD within 3 months before admission (4); discharge diagnosis along with the International Classification of Diseases, Ninth Revision (ICD-9) code; and laboratory results. Prior use of drugs (Supplementary Table S2 online) included the use of analgesics, antimicrobials, anti-epileptics, antihypertension medication, contrast media, immunosuppressants, digoxin, lipidlowering agents, and other drugs (methocarbamol and mesalazine) $(4,15)$.

\section{Statistical Analyses}

The prevalence of AKI in a group of hospitalized patients or outpatients was assessed. In the case of a small group of patients who attended an outpatient clinic several times in a day, only one visit was counted. The incident cases of AKI were analyzed at the patient level. The patients experienced multiple AKI events during the study period, and the earliest AKI event was considered as the incident case of AKI. The primary outcomes of interest were allcause, in-hospital mortality, hospital LOS, and the need for intensive care during hospitalization. Continuous data are presented as the mean (SD) or median (interquartile range (IQR), 25th-75th percentile), and categorical data as numbers and percentages. The means of baseline values were compared using unpaired $t$-tests or ANOVA tests for continuous variables and $\chi^{2}$-tests for categorical variables.

To identify any trends in CA-AKI and HA-AKI, annual prevalence changes in AKI episodes (person-visits or person-admissions) over the study period were examined using the Cochran-Armitage test for binomial outcomes (16). Comparisons between groups were carried out by using the $t$-test (or Wilcoxon test) for continuous data or Pearson $\chi^{2}$-test (or Fisher's exact test) for categorical data. We used the multivariate logistic regression model to determine the adjusted odds ratio (aOR) and $95 \%$ confidence interval (CI) to study the independent association between the occurrence of AKI and baseline characteristics. The disease conditions were identified based on the International Classification of Diseases, Ninth Revision (ICD-9-CM) code for patients, with an AKI event of the outpatient visits and hospital discharges. All analyses were carried out using SAS Enterprise Guide 5.1 version (SAS Institute, Cary, NC). All statistical tests were two-tailed, and the level of significance was $P \leq 0.05$.

\section{RESULTS \\ Prevalence and Incidence by Clinical Settings}

Over the 5-year study period, the study cohort included 87,007 patients with a total of $1,014,669$ outpatient visits, and 57,879 patients with 102,198 hospital admissions (Figure 1). The average prevalence of CANA-AKI was four cases $(95 \%$ CI, 3.8-4.21) per 1,000 outpatient visits; 17 cases (95\% CI, 15.94-18.04) per 1,000 hospital admissions for CAA-AKI; and 9.69 cases (95\% CI, 8.92-10.46) per 1,000 hospital admissions for HA-AKI. The annual rate (mean) of admission to hospital in the case of CAA-AKI increased from 13.06 (95\% CI, 11.1814.94) cases per 1,000 admissions in 2010 to 17.62 (95\% CI, 15.1-20.14) cases per 1,000 admissions in 2014 (trend test, $P<0.001$; Figure 2a). The annual rate (mean) in the case of HA-AKI declined over time, from 10.56 to 7.09 cases per 1,000 admissions (trend test, $P=0.013$ ), and the increase in the annual rate of CANA-AKI was significant (1.95-2.37 cases per 1,000 visits; trend test, $P<0.001$; Figure 2 b).

The incidence of CANA-AKI was 5.6 cases per 100 outpatient visits $(722 / 12,891)$, CAA-AKI was 12.1 cases per 100 hospital admissions (945/7,790), and HA-AKI was 13.9 cases per 100 hospital admissions (308/2,209). Among the patients with CANA-AKI, 413 were determined to have AKIN criteria stage 1 (57.2\%); $190(26.32 \%)$ to have stage 2, and $119(16.48 \%)$ to have stage 3. Among the hospitalized patients, a greater proportion of HA-AKI patients were determined to have AKIN criteria stage 3 as compared to patients with CAA-AKI $(15.91 \%$ vs. $6.46 \%, P<0.0001)$. 


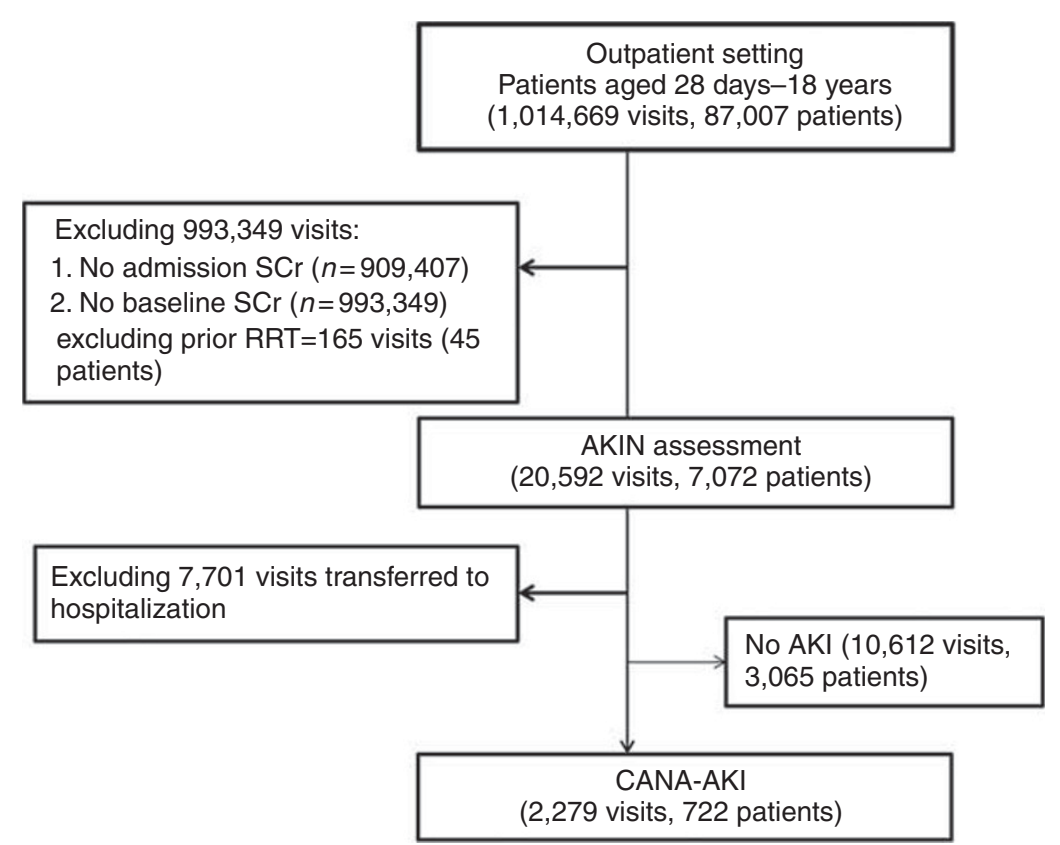

b

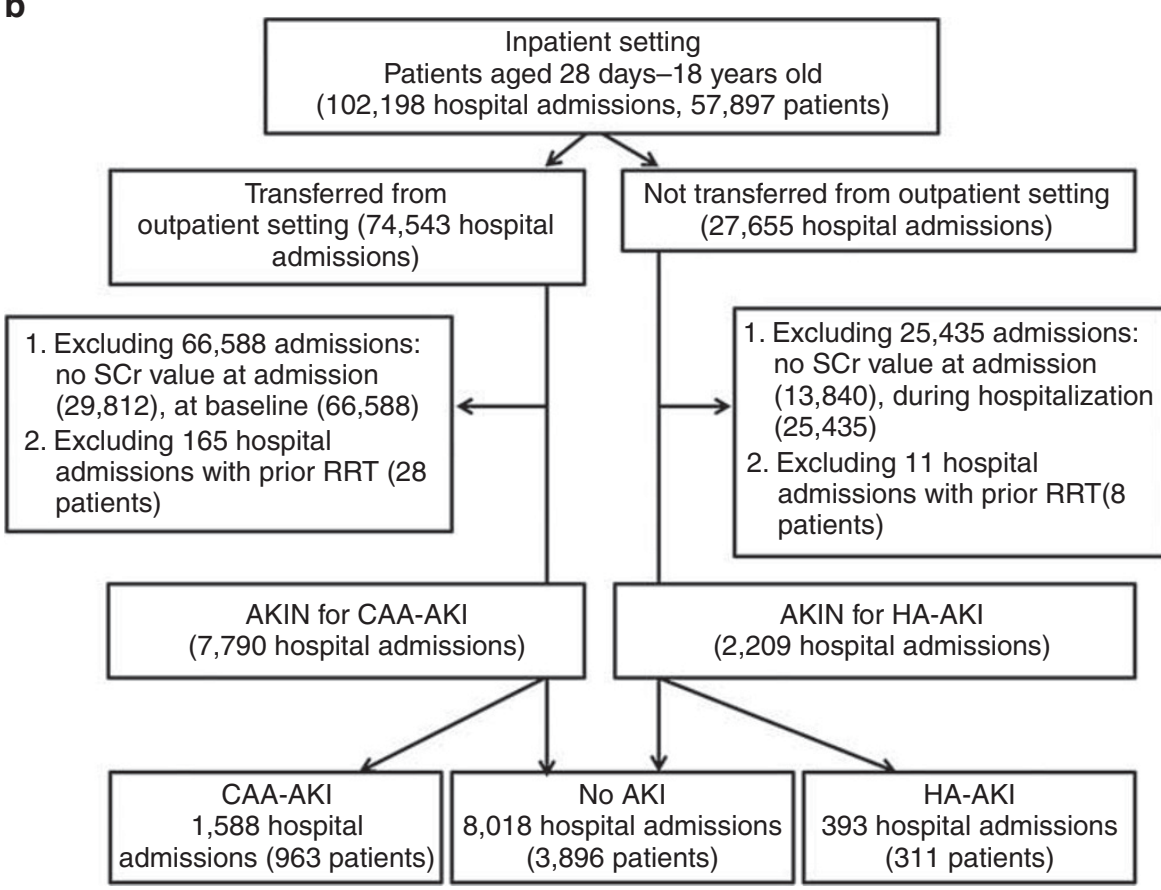

Figure 1. Description of patient selection. (a) Cases of AKI in the outpatient setting. (b) Cases of AKI in the inpatient setting. AKl, acute kidney injury.

\section{Cohort Characteristics}

Table 2 presents the demographic and clinical characteristics of the study cohort. In general, patients who developed AKI during hospitalization were younger (median 2.75, IQR 0.9110.08) than those with CAA-AKI (median 3.13, IQR 1.0110.59) and CANA-AKI (median 5.68, IQR 1.86-12.34). The number of male patients was higher in the AKI groups, and there was no apparent difference between the AKI and
non-AKI groups. When compared with patients without AKI in an outpatient setting, children with CANA-AKI had a greater incidence of pre-existing CKD (4.83\% vs. $8.86 \%$; $P<0.0001$ ), two or more hospitalizations ( $13.57 \%$ vs. $4.11 \%$, $P<0.0001)$, three or more outpatient visits $(47.09 \%$ vs. $41.08 \%, P<0.0001)$, and prior exposure to $\geq 2$ classes of nephrotoxic medications $(20.91 \%$ vs. $11.65 \%$; $P<0.001)$. Among the hospitalized patients, CAA-AKI patients had 


\section{Epidemiology and outcomes of pediatric AKI $\quad$ Articles}

Table 1. Definition of AKI groups and AKIN criteria

\begin{tabular}{|c|c|c|}
\hline AKI group & Description & AKIN definition ( $\mathrm{SCr}$ criteria) \\
\hline HA-AKI & $\begin{array}{l}\text { In inpatient setting, patient with } \mathrm{SCr} \geq 0.3 \mathrm{mg} / \mathrm{dl} \text { at first date or } \\
\text { increase } \geq 50 \% \text { of prior } \mathrm{SCr} \text { during hospitalization }\end{array}$ & $\begin{array}{l}\text { Stage 1: } \\
\text { SCr increase } \geq 0.3 \mathrm{mg} / \mathrm{dl} \text { or increase to } 1.5-2.0 \text {-folds from baseline }\end{array}$ \\
\hline CANA-AKI & $\begin{array}{l}\text { In outpatient setting (including emergency department), } \\
\text { admission } \mathrm{SCr} \geq 0.3 \mathrm{mg} / \mathrm{dl} \\
\text { Or increase } \geq 50 \% \text { of baseline } \mathrm{SCr} \\
\text { Or admission } \mathrm{SCr} \geq 4.0 \mathrm{mg} / \mathrm{dl} \text { with an acute increase of } \geq 0.5 \mathrm{mg} / \mathrm{dl} \\
\text { in } 2 \text { days } \\
\text { Or need for RRT (AKIN stage } 3 \text { ) } \\
\text { Baseline } \mathrm{SCr} \text { retrieved } \leq 3 \text { months or } 12 \text { months before index } \\
\text { admission } \\
\text { Patients did not require hospital care }\end{array}$ & $\begin{array}{l}\text { Stage 2: } \\
\text { SCr increase }>2.0-3.0 \text {-folds from baseline; } \\
\text { Stage 3: } \\
\text { SCr increase }>3.0 \text {-folds from baseline or SCr increase } \geq 4.0 \mathrm{mg} / \mathrm{dl} \\
\text { with an acute increase of } \geq 0.5 \mathrm{mg} / \mathrm{dl} \text { or need for RRT }\end{array}$ \\
\hline CAA-AKI & $\begin{array}{l}\text { Patient developed CA-AKI and subsequently admitted to hospital } \\
\text { care }\end{array}$ & \\
\hline $\begin{array}{l}\text { No AKI } \\
\text { group }\end{array}$ & $\begin{array}{l}\text { Patient did not meet with AKIN definition in outpatient or } \\
\text { inpatient setting }\end{array}$ & None of above \\
\hline
\end{tabular}

AKIN, acute kidney injury network; CANA-AKI, community-acquired AKI no admitted to hospital care; CAA-AKI, community-acquired AKI requiring admission to hospital care; HA-AKI, hospital-acquired AKI; RRT, renal replacement therapy, including dialysis and renal transplantation; SCr, serum creatinine.

a greater incidence of a history of CKD and prior use of nephrotoxic medications (two or more classes) than did the patients with HA-AKI and without AKI.

\section{Patient Outcomes}

The all-cause, in-hospital mortality rate was $1.92 \%(n=99)$ among all hospitalized patients, and 6.15\% $(n=77)$ among patients who developed AKI. The mortality rate was higher among children with AKI than among those without AKI, and it was higher among HA-AKI patients than among CAAAKI patients $(13.64 \%$ vs. $3.7 \%, P<0.0001)$. Similarly, the proportion of patients who received intensive care $(P<0.0001)$ was significantly higher among patients with AKI (HA-AKI: $10.06 \%$, CAA-AKI: $10.9 \%$ ) than among those without AKI (3.98\%). The mortality rate (AKIN stage 1, 2.86\%; stage 2, 8.09\%; and stage 3, 20.91\%; $P<0.001$ ) and the need for intensive care (AKIN stage 1, 6.8\%; stage 2, $14.71 \%$; and stage $3,21.82 \% ; P<0.0001)$ significantly increased with an increase in the severity of AKI. Hospital LOS was significantly longer among patients with HA-AKI (median 24 days, IQR 13-51) and CAA-AKI (median 7 days, IQR $5-12$ ), and increased with an increase in the severity of AKI (stage 1: median 8 days, IQR 5-15; stage 2: median 11 days, IQR 6-24; and stage 3: median 15 days, IQR 8-42; $P<0.0001$ ). The median LOS was 6 (IQR 4-10) days for hospitalized patients without AKI.

\section{Severity of AKI}

A more severe AKI episode was more likely to occur in younger patients. The youngest age at which stage 3 was identified was a median age of 1.49 years for HA-AKI, 1.97 years for CAA-AKI, and 2.26 years for CANA-AKI. The youngest age at which stage 2 was identified was a median age of 1.92 years for HA-AKI, 2.55 years for CAA-AKI, and 5.01 years for CANA-AKI. Stage 1 was identified in older children, at a median age of 7.13 years for CANA-AKI, 4.58 years for HA-AKI, and 3.57 years for CAA-AKI.
Meanwhile, the association between age and in-hospital mortality in patients with AKI was $7.36 \%$ in children aged $<2$ years (HA-AKI:CAA-AKI $=4.65: 2.71 \%$ ); the highest mortality rate noted was $9 \%$ and was observed among children aged 13 to $<18$ years (HA-AKI:CAA-AKI $=6: 3 \%$ ). The in-hospital mortality rate was $2.88 \%$ in children aged 2 to $<6$ years (HAAKI:CAA-AKI $=1.8: 1.08 \%)$ and $5.02 \%$ in children aged 6 to $<13$ years (HA-AKI:CAA-AKI = 2.7:2.32\%).

\section{Conditions Associated with AKI}

Table 3 shows the association between AKI and patient condition. In an outpatient setting, a prior diagnosis of CKD (aOR, 2.86; 95\% CI, 1.94-4.22) and recent hospitalization were associated with CANA-AKI. When compared with patients without prior hospitalization, children with two or more prior hospitalizations (aOR, 1.27; 95\% CI, 1.69-3.25) had a higher likelihood of developing CANA-AKI than did those with one prior hospitalization (aOR, 2.08; 95\% CI, 1.652.62). Likewise, prior $C K D$ and recent hospitalizations were associated with the development of AKI (including CAA-AKI and HA-AKI) during hospitalization. Prior exposure to two or more classes of medications that could be harmful for patients with impaired renal function increased the risk of developing AKI during hospitalization by $45 \%$ (aOR, 1.45; 95\% CI, 1.181.78). Of the 15 groups of diagnoses, malignancies, congenital diseases, injury, and diseases related to the circulatory system were strongly associated with AKI both in outpatient and inpatient settings $(\mathrm{aOR}>2)$, although the extent of the associations varied slightly by setting.

\section{DISCUSSION}

The present pediatric AKI study demonstrates that CA-AKI is prevalent in a practice setting. A substantial proportion of the patients treated in an inpatient setting had CA-AKI. Both CA- and HA-AKI increased the risk of mortality, but the inhospital mortality rate and health-care resource utilization 


\section{Articles | Hsu et al.}
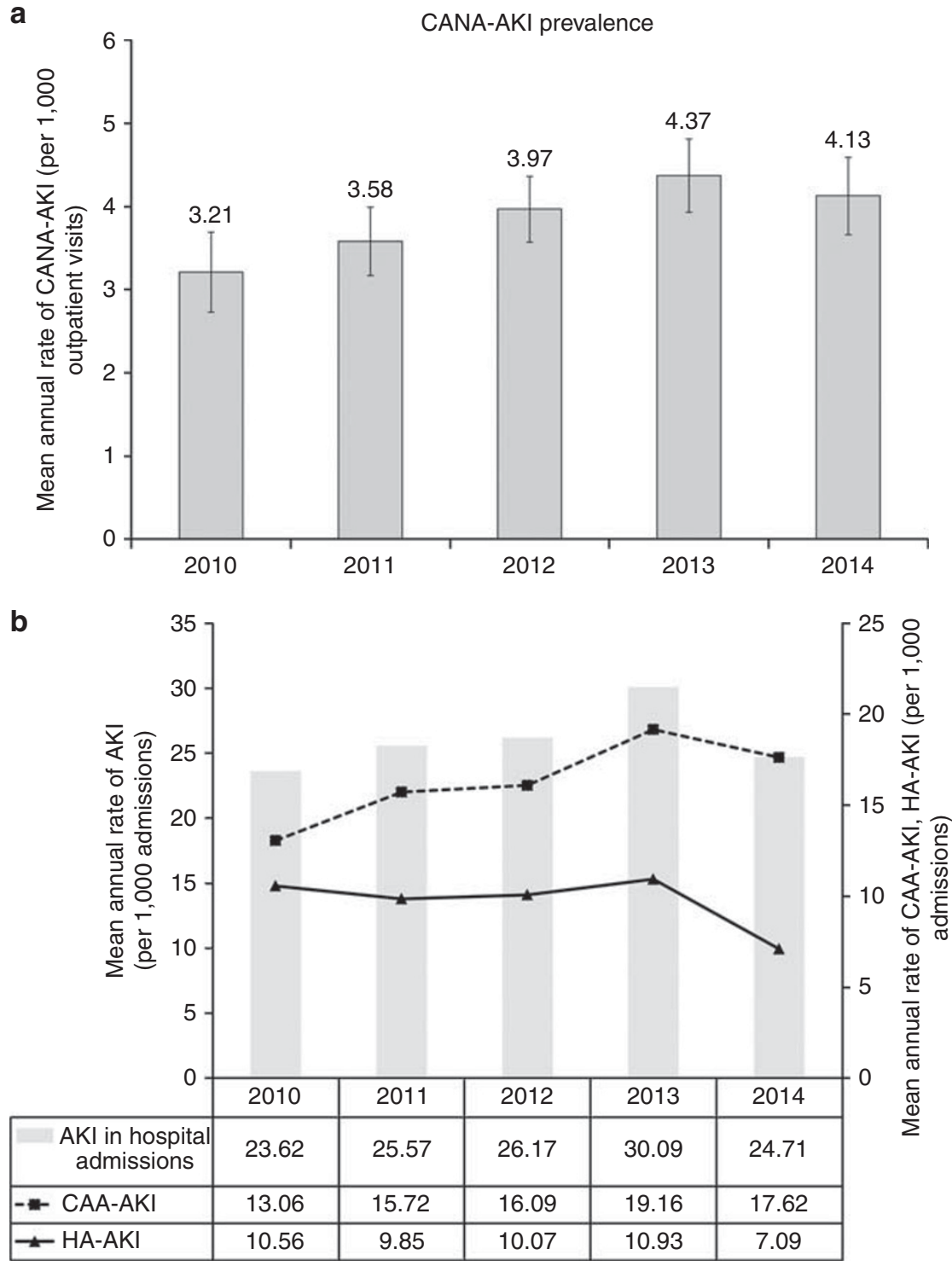

Figure 2. The annual prevalence rate of pediatric AKI. (a) AKI in the outpatient setting. Prevalence of CANA-AKI significantly increased over time (trend test, $P<0.001$ ). (b) AKI in the inpatient setting. Prevalence of AKI in inpatient setting slightly declined (trend test, $Z=-1.93, P=0.056$ ); $C A A-A K I$ significantly increased (trend test, $Z=-4.41, P<0.001$ ); HA-AKI decreased (trend test, $Z=2.59, P=0.013$ ). AKI, acute kidney injury; $C A N A-A K I$, community acquired AKI; HA-AKI, hospital-acquired AKI.

were higher among patients with HA-AKI than among CAAAKI patients.

The study results are consistent with previous studies. A study examined the incidence of AKI in hospital and community settings using NHS England AKI e-alert algorithm (17). Overall incidence of AKI was 8.9\% (4,550/50,835 adults), $61.1 \%$ with HA-AKI, $22.9 \%$ with CAA-AKI (CA-AKI admitted to hospital within 7 days), and 16\% with CANAAKI (CA-AKI not admitted within 7 days). Despite the incidence being lower than in adults, a significant burden of CA-AKI and a higher rate of CAA-AKI in our pediatric cohort were consistent in an adult population. Further, our study results suggested that the burden of pediatric AKI shifted from HA-AKI to CA-AKI as the incidence of both
CANA-AKI and CAA-AKI steadily increased over time, suggesting that the importance to raise the awareness of AKI may lead to improvements in monitoring and the preventions of patient harms.

The incidence of AKI that has been reported in noncritically ill hospitalized children in general wards was $30 \%$ from 2011 to 2012 (4) and $37.3-51.1 \%$ among all hospitalized children from 2006 to 2010 (AKIN: 37.3\%, KDIGO: $40.3 \%$, and pediatric RIFLE: $51.1 \%)$ (12). The incidence of pediatric AKI determined using the $\mathrm{SCr}$ criterion in the present $(24.6 \%)$ and previous studies $(4,12)$ was higher than that determined among intensive care patients $(1.4 \%$, or $14 / 1,000$ admissions) (1) and general hospitalized children (3.9-9.9 cases per 1,000 admissions) $(18,19)$ when discharge 
Epidemiology and outcomes of pediatric AKI | Articles

Table 2. Demographics and clinical characteristics of the study cohort

\begin{tabular}{|c|c|c|c|c|c|c|c|}
\hline & \multicolumn{3}{|c|}{ Outpatient setting } & \multicolumn{4}{|c|}{ Inpatient setting } \\
\hline & $\begin{array}{c}\text { No AKI } \\
(n=3,065)\end{array}$ & $\begin{array}{l}\text { CANA-AKI } \\
(n=722)\end{array}$ & $P$ value* & $\begin{array}{c}\text { No AKI } \\
(n=3,891)\end{array}$ & $\begin{array}{l}\text { CAA-AKI } \\
(n=945) \\
\end{array}$ & HA-AKI $(n=308)$ & $P$ value $^{* *}$ \\
\hline Age, year median (IQR) & $9.01(2.89-14.47)$ & $5.68(1.86-12.34)$ & $<0.001$ & $3.79(1.00-10.9)$ & $3.13(1.01-10.59)$ & $\begin{array}{l}2.75(0.91- \\
10.08)\end{array}$ & 0.404 \\
\hline Age group, $n$ (\%) & & & $<0.001$ & & & & 0.057 \\
\hline 2 to $<6$ & $577(18.83)$ & $177(24.52)$ & & $872(22.41)$ & $219(23.17)$ & 59 (19.16) & \\
\hline 6 to $<13$ & $863(28.16)$ & $194(26.87)$ & & 761 (19.56) & $194(20.53)$ & $65(21.1)$ & \\
\hline 13 to $<18$ & $1,005(32.79)$ & $160(22.16)$ & & 757 (19.46) & $155(16.4)$ & $45(14.61)$ & \\
\hline Male patient & $1,662(54.23)$ & $407(56.37)$ & 0.297 & $2,197(56.46)$ & $511(54.07)$ & $177(57.47)$ & 0.365 \\
\hline Baseline $\mathrm{SCr}$ & $0.5 \pm 0.34$ & $0.32 \pm 0.69$ & $<0.001$ & $0.43 \pm 0.5$ & $0.3( \pm 0.59)$ & - & \\
\hline 1 & - & $413(57.2)$ & & - & $596(63.07)$ & $139(45.13)$ & \\
\hline 2 & - & $190(26.32)$ & & - & $288(30.48)$ & $120(38.96)$ & \\
\hline 3 & - & $119(16.48)$ & & - & $61(6.46)$ & $49(15.91)$ & \\
\hline CKD diagnosis, $n$ (\%) & $148(4.83)$ & $64(8.86)$ & $<0.0001$ & $107(2.75)$ & $68(7.2)$ & $1(0.32)$ & $<0.0001$ \\
\hline $\begin{array}{l}\text { Prior use of nephrotoxic drugs, } \\
n(\%)\end{array}$ & & & $<0.001$ & & & & $<0.0001$ \\
\hline 0 & $1,715(55.95)$ & $354(49.03)$ & & $2,169(55.74)$ & $413(43.70)$ & $201(65.26)$ & \\
\hline 1 & $993(32.40)$ & $217(30.06)$ & & $1,231(31.64)$ & $325(34.39)$ & $59(19.16)$ & \\
\hline$\geq 2$ & $357(11.65)$ & $151(20.91)$ & & $491(12.62)$ & $207(21.90)$ & $48(15.58)$ & \\
\hline Prior outpatients, $n$ (\%) & & & $<0.0001$ & & & & $<0.0001$ \\
\hline$<3$ & $1,806(58.92)$ & $382(52.91)$ & & $1,769(45.46)$ & $256(27.09)$ & $142(46.1)$ & \\
\hline$\geq 3$ & $1,259(41.08)$ & $340(47.09)$ & & $2,122(54.54)$ & $689(72.91)$ & $166(53.9)$ & \\
\hline
\end{tabular}

CA-AKI, community-acquired acute kidney injury; CAA-AKI, patients with CA-AKI and requiring hospital admission; CANA-AKI, patients with CA-AKI and not requiring hospital; $\mathrm{SCr}$, serum creatinine. ${ }^{*} P$ value from Wilcoxon rank-sum test; ${ }^{* *}$ Kruskal-Wallis test.

diagnosis codes (ICD-9, 584) were used. These findings confirmed a previous adult AKI study result, which suggested that the use of ICD-9 codes in hospital discharges leads to an underestimation of the true incidence of AKI (20). We have previously shown that for adults the diagnosis code for AKI is specific for severe stage of AKI and is highly associated with poor patient outcome when RIFLE creatinine-based criteria are used (21). Although the use of the hospital discharge diagnosis provides a good clinical picture in AKI cases, it misclassifies patients who have mild-to-moderate symptoms of AKI.

Nonetheless, the previous two studies did not specify whether the AKI patients treated in inpatient was originated in the community $(4,12)$. Our analysis goes further by reporting an association between the development of AKI among pediatric patients in the community and underlying diagnoses of hematological-oncological conditions and congenital anomalies. Electrolyte and fluid disorders, renal disease, and sepsis-related conditions were common, and the incidence varied between CANA-AKI and CAA-AKI patients (Supplementary Table S3 online). In our study cohort, it is possible that the patients who were hospitalized had more severe concomitant primary diseases or higher AKI stages. Both the incidence and prevalence of CAA-AKI were higher than that of HA-AKI among hospitalized patients, and CAA-AKI was associated with a higher risk of mortality than 
Table 3. Factors associated with AKI development

\begin{tabular}{|c|c|c|}
\hline & $\begin{array}{l}\text { Outpatient setting } \\
\text { AKI; aOR }(95 \% \mathrm{Cl})\end{array}$ & $\begin{array}{l}\text { Inpatient setting } \\
\text { AKI }^{a} ; \text { aOR }(95 \% \mathrm{Cl})\end{array}$ \\
\hline \multicolumn{3}{|l|}{ Age at admission, years } \\
\hline$<2$ & $1.09(0.83,1.43)$ & $1.02(0.84,1.24)$ \\
\hline 2 to $<6$ & Ref & Ref \\
\hline 6 to $<13$ & $0.54(0.41,0.71)$ & $0.82(0.66,1.03)$ \\
\hline 13 to $<18$ & $0.39(0.30,0.52)$ & $0.68(0.53,0.86)$ \\
\hline Male (Ref = female) & $1.07(0.89,1.28)$ & $0.96(0.83,1.10)$ \\
\hline Prior CKD diagnosis & $2.86(1.94,4.22)$ & $1.54(1.06,2.23)$ \\
\hline \multicolumn{3}{|c|}{ Concomitant conditions by system $(\operatorname{Ref}=\mathrm{No})$} \\
\hline Infections & $1.52(1.10,2.09)$ & $1.36(1.15,1.61)$ \\
\hline Malignancies & $4.73(3.62,6.17)$ & $3.53(2.84,4.39)$ \\
\hline $\begin{array}{l}\text { Endocrine/metabolic } \\
\text { disorders }\end{array}$ & $1.03(0.81,1.33)$ & $1.14(0.97,1.34)$ \\
\hline Hematologic disorders & $2.31(1.62,3.30)$ & $1.38(1.14,1.68)$ \\
\hline Mental system & $0.74(0.37,1.49)$ & $0.85(0.50,1.44)$ \\
\hline CNS and sensory system & $0.88(0.67,1.17)$ & $1.48(1.22,1.79)$ \\
\hline Circulatory system & $1.44(0.97,2.13)$ & $2.50(2.00,3.11)$ \\
\hline Respiratory system & $1.01(0.83,1.24)$ & $1.26(1.08,1.47)$ \\
\hline Digestive, liver system & $2.13(1.71,2.66)$ & $1.45(1.24,1.71)$ \\
\hline Kidney-urinary, genital & $0.80(0.60,1.06)$ & $1.98(1.65,2.39)$ \\
\hline Skin system & $1.06(0.77,1.46)$ & $0.97(0.74,1.28)$ \\
\hline Musculoskeletal system & $0.79(0.52,1.23)$ & $0.83(0.57,1.22)$ \\
\hline Congenital diseases & $2.43(1.88,3.12)$ & $2.14(1.77,2.59)$ \\
\hline Fever and other symptoms & $0.89(0.68,1.16)$ & $1.09(0.84,1.42)$ \\
\hline Injury & $2.82(2.00,3.97)$ & $2.52(1.97,3.23)$ \\
\hline
\end{tabular}

Prior use of drugs with renal function concerns by class

$$
0
$$

Ref

Ref

1

$\geq 2$

$1.04(0.54,1.98)$

$1.27(0.33,4.84)$

$1.09(0.92,1.28)$

$1.45(1.18,1.78)$

Prior hospitalizations

0

1

Ref

Ref

$2.08(1.65,2.62)$

$1.22(1.03,1.45)$

$\geq 2$

$2.34(1.69,3.25)$

$4.33(3.47,5.40)$

Prior number of outpatient visits

$<3$

Ref

Ref

$\geq 3$

$1.19(0.97,1.46)$

$1.15(0.98,1.35)$

AKI, acute kidney injury; aOR, adjusted odds ratio; $95 \% \mathrm{Cl}$, confidence interval; $\mathrm{CKD}$, chronic kidney disease; CNS, central nervous system; Ref, reference.

Drugs with renal function concerns (Supplementary Table S2 online) included analgesics, antimicrobials, anti-epileptics, antihypertension, contrast media, immunosuppressants, digoxin, lipid-lowering agents, and others (methocarbamol and mesalazine).

${ }^{\mathrm{a}} \mathrm{AKI}$ in inpatient setting (including CAA-AKI and HA-AKI).

not-AKI (3.7\% vs. $1.92 \%)$; therefore, the recognition of AKI and its risk factors have important implications for CA-AKI care in an outpatient setting.

The study results also confirmed that patients who had severe stages of AKI were younger and had an increased risk of in-hospital mortality and prolonged LOS in an inpatient setting. AKI was largely associated with primary disorders, including congenital- and malignancy-related diseases and liver disease. It is important to note that the association between AKI and the high mortality rate is confounded by primary disorders, cause of hospitalization, and procedures performed and nephrotoxic medication intake during hospitalization $(1,11,22-24)$.

AKI is often preventable and reversible. Computerized provider order entry intervention $(25,26)$, electronic alerts $(27,28)$, risk assessment toolkits (29), and care bundles (30) are being increasingly implemented to facilitate early detection, improve care processes, prevent worsening of patient outcomes, and reduce health-care resource utilization in different settings. For example, identified AKI risk factors can be used to generate predictive models to screen individual patients for AKI in specific settings, such as intensive care unit (ICU) and non-ICU settings (29). Considering the increase in the incidence of CA-AKI, further studies could be useful in determining the application of risk factors identified in our analysis (patient-related underlying diseases, acute illnesses, and nephrotoxic medicines) in the development of e-alert systems and risk assessment tools for pediatric patients who are at risk for AKI in outpatient settings.

The present study has several limitations. Unlike registries' study allows prospective systematic collection of SCr and UO data (6), UO data collection is not in routine practice in both inpatient and outpatient settings. UO provides a rapid assessment of kidney function and is often used to guide fluid resuscitation in a critical care setting. The definition of AKI was based on the AKIN criteria, which assess changes in SCr levels alone, and did not include UO measurements or clinical verifications that may lead to underestimation of CAAKI due to irregular SCr testing. The AWARE study revealed that SCr criterion alone can fail to identify $67.2 \%$ AKI patients who met with UO criterion for AKI (6). Mortality was reported to be higher among patients with stage 3 AKI defined according to UO than among patients with stage 3 AKI defined according to SCr measurements (6). The results suggested that a UO criterion of $0.3 \mathrm{ml} / \mathrm{kg} / \mathrm{h}$ for $24 \mathrm{~h}$ or anuria for $12 \mathrm{~h}$ is a better predictor of advanced AKI than is the SCr criterion; further, it is likely that the incidence of stage 3 HAAKI was underestimated in the previous and the present study that used the SCr criteria alone.

Because the data were retrieved from a single tertiary-care hospital in Taiwan, the AKI severity and resource use may not be generalizable to other practice settings; however, the association between pediatric AKI and health-care service utilization is consistent with Western populations. In addition, recurrent AKI, long-term mortality, CKD development, and renal function progression were not evaluated in the present study, but should be considered in future research efforts $(8,9)$.

\section{Conclusions}

The present study emphasizes the need for monitoring $\mathrm{SCr}$ values in children in an outpatient setting. A careful 


\section{Epidemiology and outcomes of pediatric AKI $\mid$ Articles}

assessment of primary disease conditions, clinical circumstances, and modifiable risk factors such as nephrotoxic medication and fluid supplementation is imperative for the early identification and proper management of AKI in an outpatient setting in order to prevent the worsening of AKI in at-risk pediatric populations.

\section{SUPPLEMENTARY MATERIAL}

Supplementary material is linked to the online version of the paper at http://www.nature.com/pr

\section{ACKNOWLEDGMENTS}

We thank Ying-Jen Hsu and the SAS Working Group in the Department of Management of Information Systems for EMRs acquisition; Han-Chun Huang for helping with presentation of results in the tables and figures; Dai-Hua Liu in the Medical Record Services for disease coding assistance; and Yu-Shao Chiang and Tsai-Ling Chao in the Division of Laboratory Medicine for help with laboratory result validation in Kaohsiung Chang Gung Memorial Hospital.

\section{STATEMENT OF FINANCIAL SUPPORT}

This work was supported by the National Health Research Institute (NHRIEX104-10227PC) and Kaohsiung Chang Gung Memorial Hospital (CMRPG8C1302) in Taiwan. The funding sources were not involved in the study design or conduct; collection, management, analysis, or interpretation of the data; approval of the manuscript; or the decision to submit the manuscript for publication.

Disclosure: The authors declare no conflict of interest.

\section{REFERENCES}

1. Chang JW, Jeng MJ, Yang LY, et al. The epidemiology and prognostic factors of mortality in critically ill children with acute kidney injury in Taiwan. Kidney Int 2015;87:632-9.

2. Olowu WA, Adefehinti O, Bisiriyu AL. Hospital-acquired acute kidney injury in critically ill children and adolescents. Saudi J Kidney Dis Transpl 2012;23:68-77.

3. Alkandari O, Eddington KA, Hyder A, et al. Acute kidney injury is an independent risk factor for pediatric intensive care unit mortality, longer length of stay and prolonged mechanical ventilation in critically ill children: a two-center retrospective cohort study. Crit Care 2011;15:R146.

4. McGregor TL, Jones DP, Wang L, et al. Acute kidney injury incidence in noncritically ill hospitalized children, adolescents, and young adults: a retrospective observational study. Am J Kidney Dis 2015;67:384-90.

5. Zappitelli M, Moffett BS, Hyder A, Goldstein SL. Acute kidney injury in non-critically ill children treated with aminoglycoside antibiotics in a tertiary healthcare centre: a retrospective cohort study. Nephrol Dial Transplant 2011;26:144-50.

6. Kaddourah A, Basu RK, Bagshaw SM, Goldstein SL. Epidemiology of acute kidney injury in critically ill children and young adults. N Engl J Med 2017;376:11-20.

7. Hoste EA, Bagshaw SM, Bellomo R, et al. Epidemiology of acute kidney injury in critically ill patients: the multinational AKI-EPI study. Intensive Care Med 2015;41:1411-23.

8. Coca SG, Singanamala S, Parikh CR. Chronic kidney disease after acute kidney injury: a systematic review and meta-analysis. Kidney Int 2012;81: 442-8.

9. Goldstein SL. Acute kidney injury in children and its potential consequences in adulthood. Blood Purif 2012;33:131-7.
10. Jha V, Parameswaran S. Community-acquired acute kidney injury in tropical countries. Nat Rev Nephrol 2013;9:278-90.

11. Cerda J, Bagga A, Kher V, Chakravarthi RM. The contrasting characteristics of acute kidney injury in developed and developing countries. Nat Clin Pract Nephrol 2008;4:138-53.

12. Sutherland SM, Byrnes JJ, Kothari M, et al. AKI in hospitalized children: comparing the pRIFLE, AKIN, and KDIGO definitions. Clin J Am Soc Nephrol 2015;10:554-61.

13. Sawhney S, Fluck N, Marks A, et al. Acute kidney injury-how does automated detection perform? Nephrol Dial Transplant 2015;30:1853-61.

14. Mehta RL, Kellum JA, Shah SV, et al. Acute Kidney Injury Network: report of an initiative to improve outcomes in acute kidney injury. Crit Care 2007;11:R31.

15. Inker LA, Astor BC, Fox CH, et al. KDOQI US commentary on the 2012 KDIGO clinical practice guideline for the evaluation and management of CKD. Am J Kidney Dis 2014;63:713-35.

16. Agresti A. Categorical Data Analysis. New York, NY: John Wiley and Sons, 2002.

17. Sawhney S, Fluck N, Fraser SD, et al. KDIGO-based acute kidney injury criteria operate differently in hospitals and the community-findings from a large population cohort. Nephrol Dial Transplant 2016;31:922-9.

18. Sutherland SM, Ji J, Sheikhi FH, et al. AKI in hospitalized children: epidemiology and clinical associations in a national cohort. Clin J Am Soc Nephrol 2013;8:1661-9.

19. Vachvanichsanong P, Dissaneewate P, Lim A, McNeil E. Childhood acute renal failure: 22-year experience in a university hospital in southern Thailand. Pediatrics 2006;118:e786-91.

20. Waikar SS, Wald R, Chertow GM, et al. Validity of international classification of diseases, ninth revision, clinical modification codes for acute renal failure. J Am Soc Nephrol 2006;17:1688-94.

21. Hsu CN, Lee CT, Su CH, et al. Incidence, outcomes, and risk factors of community-acquired and hospital-acquired acute kidney injury: a retrospective cohort study. Medicine 2016;95:e3674.

22. Chang JW, Tsai HL, Wang HH, Yang LY. Outcome and risk factors for mortality in children with acute renal failure. Clin Nephrol 2008;70: $485-9$.

23. Jang WS, Kim WH, Choi K, et al. Incidence, risk factors and clinical outcomes for acute kidney injury after aortic arch repair in paediatric patients. Eur J Cardiothorac Surg 2014;45:e208-14.

24. MacDonald C, Norris C, Alton GY, Urschel S, Joffe AR, Morgan CJ. Acute kidney injury after heart transplant in young children: risk factors and outcomes. Pediatr Nephrol 2015;31:671-8.

25. Goldstein SL, Kirkendall E, Nguyen H, et al. Electronic health record identification of nephrotoxin exposure and associated acute kidney injury. Pediatrics 2013;132:e756-67.

26. McCoy AB, Waitman LR, Gadd CS, et al. A computerized provider order entry intervention for medication safety during acute kidney injury: a quality improvement report. Am J Kidney Dis 2010;56:832-41.

27. NHS England. Patient safety alert on standardising the early identification of Acute Kidney Injury, 9 June 2014. (https://www.england.nhs.uk/ 2014/06/psa-aki/) Accessed 28 June 2017.

28. Wilson FP, Shashaty M, Testani J, et al. Automated, electronic alerts for acute kidney injury: a single-blind, parallel-group, randomised controlled trial. Lancet 2015;385:1966-74.

29. Wang L, McGregor TL, Jones DP, et al. Electronic health record-based predictive models for acute kidney injury screening in pediatric inpatients. Pediatr Res 2017;82:465-73.

30. Kolhe NV, Reilly T, Leung J, et al. A simple care bundle for use in acute kidney injury: a propensity score-matched cohort study. Nephrol Dial Transplant 2016;31:1846-54. 